\title{
ANÁLISIS COMPRENSIVO DE LOS TRAUMATISMOS TORÁCICOS PENETRANTES ATENDIDOS EN HOSPITAL PADRE HURTADO DE SANTIAGO*
}

\author{
Drs. Marcelo Parra N. ${ }^{1}$, José Ortega S. ${ }^{1}$, Raúl Berrios S. ${ }^{1}$, \\ Daniela Dávila W. ${ }^{2}$, Ángela Santa María M. ${ }^{2}$
}

1 Equipo de Cirugía de Tórax, Hospital Padre Hurtado. Facultad de Medicina Clínica Alemana-Universidad del Desarrollo.

2 Interna Cirugía, Facultad de Medicina Clínica Alemana-Universidad del Desarrollo. Santiago-Chile.

\begin{abstract}
Penetrating chest trauma

Objectives: Penetrating chest trauma (PCT) is a life threatening condition that challenges emergency surgeons daily. The aim of this study is to make an epidemiological characterization of these patients, and secondarily analyze their treatment and outcome. Methods: A retrospective analysis of a series of consecutive patients experiencing PCT who presented at our hospital, was performed from 1t May 2009 to $30^{\text {th }}$ April 2013. Results: Of 274 consecutive patients who presented to the emergency department (ED) with PCT, 257 (94\%) were male and $17(6 \%)$ were female. The median age was 26 (range 15-66) years. Stab wounds (SW) accounted for $185(68 \%)$ of the injuries, and 80 (29\%) suffered from gunshot wounds (GSW). As first treatment, chest tube drainage was performed in $229(84 \%)$ patients, emergent thoracotomy in $21(8 \%)$ and observation, in $13(5 \%) .26(9 \%)$ patients died: 21 (81\%) from GSW and 4 (15\%) with SW P $<0.0001 ; 20$ (77\%) had heart or thoracic great vessels involvement. Thoracic complications occurred in $30(12 \%)$ patients. There was no mortality associated with thoracic complications. The median hospital stay was 4 days. Conclusions: PCT is frequent in our hospital compared with historical series. The majority of the patients who died had cardiac or thoracic great vessels involvement due to GSW. Therefore, healthcare improvements are needed to reduce mortality in this group of patients.
\end{abstract}

Key words: Thoracic trauma, penetrating trauma, chest injuries.

\section{Resumen}

Objetivos: Los traumatismos torácicos penetrantes (TTP) son graves y desafían diariamente a los cirujanos de urgencia. El objetivo de este estudio es realizar una caracterización epidemiológica de los pacientes con TTP, y como objetivo secundario analizar el tratamiento efectuado y su evolución. Métodos: Se realizó

\footnotetext{
Los autores no refieren conflictos de interés.

Correspondencia: Dr. Marcelo Parra N. mfparra@gmail.com
}

*Recibido el 2 de septiembre de 2013 y aceptado para publicación el 29 de diciembre de 2013. 
un estudio retrospectivo de los pacientes atendidos en el Hospital Padre Hurtado de Santiago que presentaron un TTP desde el 1 de mayo de 2009 hasta el 30 de abril de 2013. Resultados: 274 pacientes que se consultaron al servicio de urgencia con un TTP, 257 (94\%) eran hombres y 17 (6\%) eran mujeres. La mediana de edad fue 26 (15-66) años. Lesiones por arma blanca 185 (68\%), y 80 (29\%) fueron por arma de fuego. Como primer tratamiento la pleurostomía fue realizada en 229 (84\%) pacientes. La toracotomía de emergencia fue realizada en $21(8 \%)$ pacientes. No se realizó tratamiento y solo observación en $13(5 \%)$ de los casos. 26 $(9 \%)$ de los pacientes murieron, $21(81 \%)$ fueron consecuencia de lesiones por arma de fuego y $4(15 \%)$ por arma blanca $\mathrm{P}<0,0001,20$ (77\%) tenían lesiones cardíacas o de grandes vasos torácicos. $30(12 \%)$ pacientes presentaron complicaciones torácicas. No hubo mortalidad asociada a complicaciones torácicas. La mediana de días de hospitalización fue 4. Conclusiones: El TTP es frecuente en nuestro hospital comparado con series históricas. La mayoría de los pacientes fallecidos presentaban TTP por arma de fuego con lesiones cardíacas o de grandes vasos torácicos. Se precisan mejoras asistenciales en este grupo para disminuir su mortalidad.

Palabras clave: Trauma Torácico, trauma penetrante, lesiones torácicas.

\section{Introducción}

Los traumatismos torácicos penetrantes (TTP) son una condición que frecuentemente pone en riesgo la vida del paciente y son muchas veces un desafío para los cirujanos de urgencia. El servicio de Emergencia Adulto del Hospital Padre Hurtado de Santiago comienza a funcionar a finales de abril del 2009. Principalmente atiende a pacientes de las comunas de La Granja, La Pintana y San Ramón con una población estimada de 417.511 habitantes según el censo de $2002^{1}$. El objetivo principal de este estudio es realizar una descripción epidemiológica de los pacientes que presentaron un traumatismo torácico penetrante y secundariamente analizar su tratamiento y evolución.

\section{Material y Método}

Se realizó un análisis retrospectivo de una serie de casos de TTP consecutivos que se presentaron al servicio de urgencia adulto del Hospital Padre Hurtado desde el 1 de mayo de 2009 hasta el 30 de abril de 2013. Se analizaron variables demográficas (edad, sexo, tipo de traumatismo penetrante), presentación clínica imágenes solicitadas, lesiones asociadas, procedimientos realizados, hallazgos intraoperatorios, requerimientos de transfusiones y evolución (tiempo de hospitalización, necesidad de UCI, complicaciones y mortalidad). Los TTP bilaterales fueron analizados como un paciente único y el tiempo de días con drenaje pleural se consideró desde la instalación hasta el retiro del último tubo pleural. Las lesiones pulmonares se clasificaron según el Score de Moore ${ }^{2}$. Se excluyeron los pacientes menores de 15 años, ya que estos son manejados por un servicio independiente. Así mismo no se incluyeron pacientes que presentaron paro cardiorrespi- ratorio no presenciado o maniobras de reanimación mayores a 10 minutos antes de la llegada al servicio de emergencias. La causa de fallecimiento de los pacientes no intervenidos se obtuvo del informe de las autopsias. La asociación de las variables cualitativas se realizó mediante la prueba exacta de Fisher.

\section{Resultados}

En el período estudiado consultaron 274 pacientes con TTP. En promedio consultó 1 TTP cada 6 días. El 93,80\% (257) correspondieron a hombres y el 6,20\% (17) a mujeres. La mediana de la edad fue de 26 años con un rango de 15-66 años. La incidencia de traumatismo torácico según mecanismo de origen (Arma blanca, Arma de fuego u otro) se describe en la Figura 1. Los pacientes catalogados como Otro corresponden a: 3 por TTP con fierros en relación a caídas de altura y accidentes de tránsito, 3 con vidrios, 1 con un clavo, 1 con un alfiler y por último 1 con un tenedor.

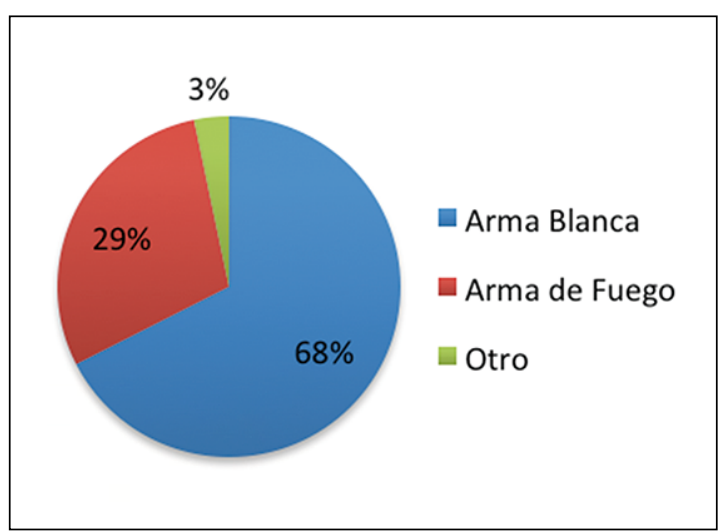

Figura 1. Mecanismo del traumatismo penetrante torácico. 


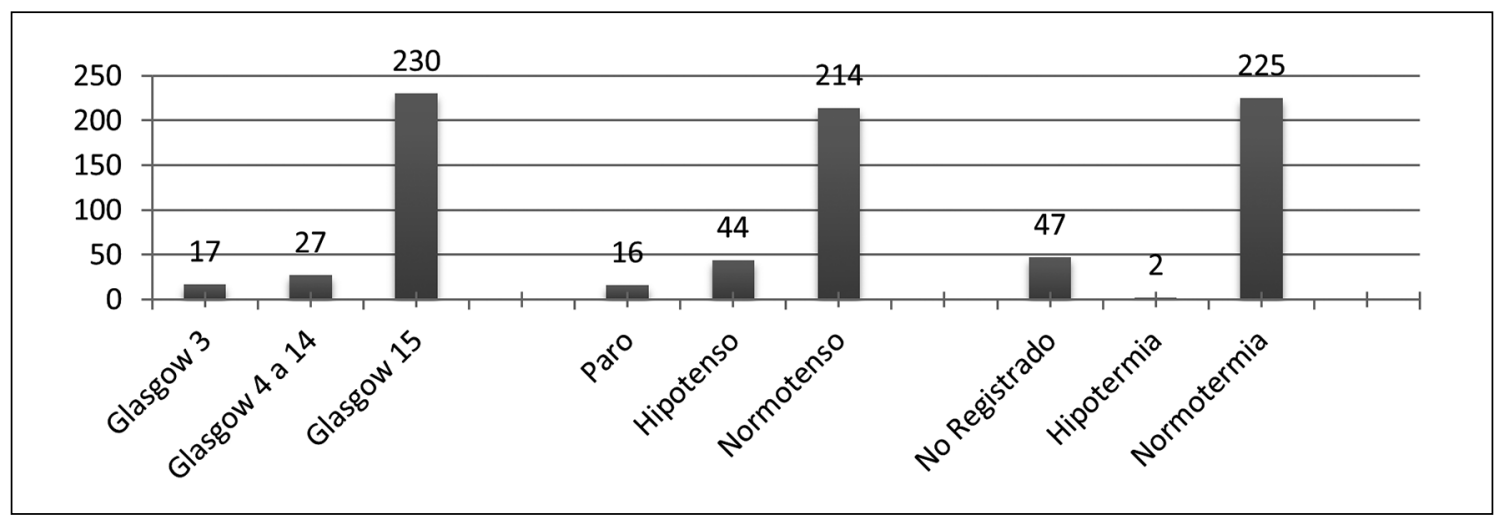

Figura 2. Condición clínica de los pacientes al ingreso al Servicio de Emergencias.

Un 9,49\% (26) de los TTP fueron bilaterales. Las condiciones generales de los pacientes al ingreso graficados en la Escala de Coma de Glasgow, presión arterial y temperatura se describen en el la Figura 2.

Se analizaron las imágenes solicitadas a los pacientes con TTP al ingreso del servicio de Emergencias. A $166(60,58 \%)$ de los pacientes sólo se le solicitó una radiografía de tórax, a $70(25,55 \%)$ de los pacientes se les solicitó una radiografía mas una TC de tórax, a $2(0,73 \%)$ de los pacientes solo se les solicitó una TC de tórax y finalmente a $36(13,14 \%)$ de los pacientes no se les realizó imágenes en el servicio de emergencias.

De acuerdo a la evaluación inicial con o sin imágenes se realizó un tratamiento inicial que está descrito en la Tabla 1. En forma inmediata o diferida algunos pacientes requirieron un tratamiento secundario el cual está descrito en la Tabla 2. Los pacientes que fueron explorados quirúrgicamente presentaban los siguientes hallazgos: lesiones cardíacas $28(10,22 \%)$, lesiones de diafragma 26 $(9,49 \%), 11(4,01 \%)$ con lesiones de grandes vasos, y finalmente lesiones de pared $2(0,73 \%)$. En relación a las lesiones pulmonares encontradas (en TC o en explorados quirúrgicamente) según el Score de Moore $^{2}$ descrito en la Tabla 3, 194 (92\%) presentaban una lesión nivel II-Laceración, 15 (7\%) tenían un score III-Hematoma y finalmente en $3(1 \%)$ se encontró una lesión IV-Vascular. No hubo cirugías de control de daño torácicas, si hubo control de daño abdominal en pacientes con traumatismo tóracoabdominal asociado.

Todos los traumatismos penetrantes torácicos presentaban una combinación de hemo-neumotórax variando la proporción de ambos. No se puede clasificar a esta serie de acuerdo a la magnitud del
Tabla 1. Tratamiento inicial del paciente con un traumatismo torácico penetrante

\begin{tabular}{|lrr|}
\hline Tratamiento inicial & & \\
\hline Pleurostomía & 229 & $(83,58 \%)$ \\
\hline Toracotomía & 21 & $(7,66 \%)$ \\
\hline Videotoracoscopía (VTC) & 1 & $(0,36 \%)$ \\
\hline Fallece sin tratamiento & 4 & $(1,46 \%)$ \\
Observación & 19 & $(6,93 \%)$ \\
Total tratamiento inicial & 274 & $(100 \%)$ \\
\hline
\end{tabular}

Tabla 2. Tratamiento secundario del paciente con un traumatismo torácico penetrante

\begin{tabular}{|lrr|}
\hline Tratamiento secundario & & \\
\hline Sólo tratamiento inicial & 199 & $(72,63 \%)$ \\
Toracotomía & 23 & $(8,39 \%)$ \\
$\begin{array}{l}\text { Reparación diafragmática desde } \\
\text { el abdomen }\end{array}$ & 14 & $(5,11 \%)$ \\
Observación & $13^{*}$ & $(4,74 \%)$ \\
Pleurostomía (primera diferida o segunda) & 12 & $(4,38 \%)$ \\
Videotoracoscopía (VTC) & 7 & $(2,55 \%)$ \\
\hline $\begin{array}{l}\text { Fallecidos sin tratamiento inicial } \\
\text { ni secundario }\end{array}$ & 4 & $(1,46 \%)$ \\
Cierre pared (rotura intercostales) & 1 & $(0,36 \%)$ \\
Pericardiocentesis & 1 & $(0,36 \%)$ \\
Total tratamiento secundario & 274 & $(100 \%)$ \\
\hline
\end{tabular}

*Sin tratamiento $1^{\circ}$ ó $2^{\circ}$. 
Tabla 3. Escala de lesión pulmonar de Moore*

\begin{tabular}{|c|c|}
\hline Grado & Descripción de la lesión \\
\hline \multicolumn{2}{|l|}{$\mathbf{I}$} \\
\hline Contusión & Unilateral, $<1$ lóbulo \\
\hline \multicolumn{2}{|l|}{ II } \\
\hline Contusión & Unilateral, 1 lóbulo \\
\hline Laceración & Neumotórax simple \\
\hline \multicolumn{2}{|l|}{ III } \\
\hline Contusión & Unilateral, > 1 lóbulo \\
\hline Laceración & $\begin{array}{l}\text { Fuga aérea persistente }(>72 \mathrm{~h}) \text {, fuga } \\
\text { aérea de la vía aérea distal }\end{array}$ \\
\hline Hematoma & Intraparenquimatoso, no expansivo \\
\hline \multicolumn{2}{|l|}{ IV } \\
\hline Laceración & Fuga aérea mayor (lobar o segmentaria) \\
\hline Hematoma & Intraparenquimatoso expansivo \\
\hline Vascular & $\begin{array}{l}\text { Lesión de una rama primaria intrapul- } \\
\text { monar }\end{array}$ \\
\hline \multicolumn{2}{|l|}{$\mathbf{V}$} \\
\hline Vascular & Lesión de un vaso hiliar \\
\hline \multicolumn{2}{|l|}{ VI } \\
\hline Vascular & $\begin{array}{l}\text { Total, transección contenida del hilio } \\
\text { pulmonar }\end{array}$ \\
\hline
\end{tabular}

Avanzar un grado para lesiones pulmonares bilaterales. *Adaptado de Moore EE, Malangoni MA, Cogbill TH, Shackford SR, Champion HR, Jurkovich GJ, McAninch JW, Trafton PG. Organ injury scaling. IV: Thoracic vascular, lung, cardiac, and diaphragm. J Trauma 1994;36:299-300.

hemo y/o neumotórax, por que los métodos de imágenes diagnósticos son diferentes, no todos son explorados, y a veces el tratamiento es previo a las imágenes (lesiones por arma de fuego donde la conducta fue la instalación de una pleurostomía antes de la imagen diagnóstica).

La mortalidad en esta serie fue de 26 pacientes equivalentes a un $9,49 \%$. Veinticinco fallecieron en el servicio de emergencia o durante el acto quirúrgico de ingreso. Sólo 1 paciente falleció en forma diferida tras múltiples complicaciones sépticas secundarias a una fístula duodenal de alto débito 306 días después del ingreso y sin conflicto torácico. De los pacientes fallecidos el 73,08\% (19) de ellos presentaban lesiones torácicas aisladas (cardíacas y grandes vasos). En la Tabla 4 se muestra la lesión que ocasionó la muerte del paciente. Al analizar el subgrupo de los pacientes fallecidos un $80,77 \%$ (21) correspondieron a lesiones por arma de fuego, $15,38 \%$ (4) por heridas por arma blanca significativamente menor con un $\mathrm{p}<0,0001$ y finalmente
Tabla 4. Causas de mortalidad de los pacientes

\begin{tabular}{|lr|}
\hline Causas de mortalidad & \\
\hline Lesión cardíaca & $15(57,69 \%)$ \\
\hline Lesión grandes vasos torácicos & $5(19,23 \%)$ \\
\hline Lesión grandes vasos abdominales & $4(15,38 \%)$ \\
Sepsis (complicación fístula duodenal) & $1 \quad(3,85 \%)$ \\
$\begin{array}{l}\text { Hemotórax masivo sin lesión letal } \\
\text { identificable }\end{array}$ & $1 \quad(3,85 \%)$ \\
Total fallecimientos & $26 \quad(100 \%)$ \\
\hline
\end{tabular}

$3,85 \%$ (1) caso de rotura de arteria subclavia por un fierro en un accidente automovilístico. En 15 pacientes se encontró lesión cardíaca por arma de fuego, 9 de estos ingresaron en paro cardiorespiratorio, 5 hipotensos y 1 normotenso. En éste subgrupo (lesiones cardiacas por arma de fuego) a 11 pacientes se les realizó una toracotomía de emergencia en box de reanimación de urgencia sin éxito. A los 4 pacientes restantes se les realizó una toracotomía urgente en pabellón logrando supervivencia. Sólo hubo un paciente con lesión por AF con afectación cardíaca en nuestra serie con un taponamiento, el resto había afectación pericárdica contraponiéndose al taponamiento. Un 6,20\% es decir 17 pacientes ingresaron en paro cardiorrespiratorio al servicio (presenciado con menos de 10 minutos de maniobras) y no sobrevivieron. Un $83,46 \%$ (227) de los pacientes no requirió transfusiones.

De los 274 pacientes incluidos en este estudio fallecieron 25 en el servicio de emergencia o durante el acto quirúrgico de ingreso; hubo 2 pérdidas de seguimiento de la serie que se trasladaron a otro centro hospitalario para manejo de lesiones asociadas por neurocirugía una vez estabilizado el traumatismo torácico. Dado lo anterior se logró un seguimiento completo a 247 pacientes. La mediana de días de tubo pleural fue de 3 días rango $0-48$. A un $83,00 \%$ (205) de los pacientes se les administró tratamiento antibiótico profiláctico. Un 12,15\% (30) de los pacientes estuvo hospitalizado en la Unidad de Paciente Crítico. UCI-UTI. La mediana de hospitalización fue de 4 días (rango 1 y 306). Un 12,15\% (30) de los pacientes presentó una complicación torácica las cuales se describen en la Tabla 5. A un 63,33\% (19) de los pacientes complicados se les que realizó un procedimiento mínimamente invasivo para tratarlo (nueva pleurostomía o Videotoracoscopía). No hubo mortalidad en los pacientes que presentaron complicaciones torácicas. Las lesiones asociadas están tabuladas en Tabla 6. 
Tabla 5. Complicaciones torácicas de los pacientes con TTP

\begin{tabular}{|lrc|}
\hline Complicaciones torácicas & 8 & $(3,24 \%)$ \\
Hemotórax residual & 7 & $(2,83 \%)$ \\
Neumonía intrahospitalaria & 4 & $(1,62 \%)$ \\
Empiema & 4 & $(1,62 \%)$ \\
Recurrencia del neumotórax & 2 & $(0,81 \%)$ \\
Sangrado $2^{\circ}$ tiempo & 1 & $(0,40 \%)$ \\
Infarto agudo al miocardio & 1 & $(0,40 \%)$ \\
Hemopericardio & 1 & $(0,40 \%)$ \\
Infección herida operatoria & 1 & $(0,40 \%)$ \\
Hernia diafragmática & 1 & $(0,40 \%)$ \\
Osteomielitis clavicular & 30 & $(12,5 \%)$ \\
Total & & \\
\hline
\end{tabular}

Tabla 6. Lesiones asociadas de los pacientes con TTP

\begin{tabular}{|c|c|}
\hline \multicolumn{2}{|l|}{ Lesiones asociadas } \\
\hline Sin lesiones & $218(79,56 \%)$ \\
\hline Abdominal & $40(14,60 \%)$ \\
\hline Columna y extremidades & $9(3,28 \%)$ \\
\hline Cerebro y sistema nervioso central & $5(1,82 \%)$ \\
\hline Facial y cervical & $2(0,73 \%)$ \\
\hline Total & $274 \quad(100 \%)$ \\
\hline
\end{tabular}

\section{Discusión}

Al realizar un análisis descriptivo de los TTP destaca en nuestro centro una incidencia anual elevada al compararla con escasas series históricas publicadas, Gómez G. y cols. ${ }^{3}$ el año 2009, presentan una serie de 36 pacientes con heridas penetrantes cardíacas operadas en un período de 24 años y del mismo modo, Valenzuela M. y cols. ${ }^{4}$ el 2003, presentan 163 traumatismos torácicos donde 131 son TTP en un período de 3,5 años. La mayoría de los TTP en nuestro centro afectaron a pacientes jóvenes y de sexo masculino no diferenciándose de lo publicado por otros centros ${ }^{3-6}$.

Si bien, las lesiones por arma blanca fueron mas frecuentes, destaca un elevado número de lesiones por arma de fuego en este estudio. En nuestra serie 3 de cada 10 de los pacientes con TTP fueron por arma de fuego comparado con la serie de Gómez
G y cols. ${ }^{3}$ del 2009 y por Valenzuela M y cols. ${ }^{4}$ del 2003, en donde 9 de cada 10 pacientes con TTP fue por arma blanca.

La gran mayoría de los pacientes que fallecieron presentaban lesiones por arma de fuego con afectación cardíaca y/o grandes vasos torácicos. Cuando la lesión torácica fue por arma de fuego la mortalidad fue significativamente mayor. No hubo supervivencia entre los pacientes que ingresaron en paro cardiorrespiratorio presenciado con menos de 10 minutos de maniobras de reanimación donde al igual que la literatura publicada la sobrevida es muy baja e incluso anecdótica ${ }^{7-9}$.

El diagnóstico de TTP es clínico, sin embargo, ante dudas diagnósticas, sospecha de lesiones asociadas y seguimiento de estos pacientes en un alto porcentaje se solicitó rayos X y TC. Algunos autores promueven en forma rutinaria el uso de la TC incluso en casos que la radiografía está normal ${ }^{10,11}$.

El drenaje pleural aislado fue el tratamiento más frecuente e incluso único tratamiento realizado a los pacientes que presentan un traumatismo torácico penetrante. Este manejo es justificado por gran cantidad de artículos publicados ${ }^{12-14}$. En un porcentaje bajo se realizó solo observación, esto en contexto de lesiones mediastínicas que no presentaron complicaciones en el seguimiento o en casos con neumo y/o hemotórax laminares. Las lesiones pulmonares excepcionalmente fueron un problema en la serie estudiada (3 casos de Lesión tipo IV-Vascular), no hubo fugas aéreas persistentes en nuestros pacientes que requiriesen un manejo específico.

En esta serie fue muy pequeña la proporción de manejo por videotoracoscopía (VTC) como enfrentamiento inicial del paciente con TTP. Esto podría estar en relación a la experiencia de nuestro servicio de emergencia y también a que el recurso a veces no siempre está disponible durante los turnos. Por el contrario, gran parte de las complicaciones sí se resolvieron en forma mínimamente invasiva. Constantemente se están publicando series que avalan el uso de VTC en los pacientes con trauma torácico tanto en su enfoque inicial como para el manejo de las complicaciones. Así, Ahmed y cols. ${ }^{15}$, trataron a 3 de cada 10 pacientes con VATS demostrando un menor tiempo de hospitalización, menor requerimiento camas de paciente crítico, menor requerimiento de toracotomías y por su parte Goodman y cols ${ }^{16}$, donde demuestran la seguridad de la técnica en pacientes hemodinámicamente estables.

La mayoría de los pacientes requirieron una cama básica y pocos días con drenaje pleural. Un gran porcentaje de los pacientes recibió antibiótico profilaxis, asumiendo que la herida penetrante torácica es una herida sucia. Estudios demuestran con buena 
evidencia que su uso está indicado ${ }^{17}$. No se puede saber con este estudio si hay relación entre la profilaxis antibiótica y la baja incidencia de empiemas en nuestra serie.

La complicación asociada más frecuente en tórax fue la presencia de hemotórax, seguida de neumonía intrahospitalaria y recurrencia del neumotórax las cuales fueron resueltas en su mayoría de forma mínimamente invasiva sin aumentar la mortalidad. De las lesiones asociadas extra torácicas las mas frecuentes fueron las lesiones abdominales, seguidas por las lesiones músculo esqueléticas, lo que también está en concordancia con lo publicado en la literatura internacional. ${ }^{17}$ Efectivamente, en la serie de Mandal y cols $^{18}$, donde un $46 \%$ de los pacientes tenían lesiones abdominales asociadas.

Hubo una escasa pérdida de seguimiento de los pacientes ya que algunos de estos fueron trasladados a otro centro para manejo por neurocirugía una vez estabilizado el cuadro torácico por lo cual tampoco debiese afectar significativamente los resultados globales.

\section{Conclusiones}

El TTP es frecuente en nuestro hospital comparado con series históricas de otros centros nacionales. Sin embargo, faltan mas estudios nacionales recientes de diferentes centros y así poder ilustrar con mayor claridad esta realidad.

A pesar de los sesgos que puedan presentar los estudios retrospectivos, este trabajo nos ilustra un problema de salud local justificando la realización de mas estudios idealmente prospectivos.

Finalmente, se debe buscar la forma de intervenir para reducir la mortalidad en el subgrupo de pacientes que presentaba un TTP por arma de fuego con lesiones cardíacas o de grandes vasos torácicos, buscando como reducir los tiempos de rescate y realizar entrenamiento continuo del personal de salud local enfrentado a estos pacientes.

\section{Agradecimientos}

Al Dr. Ramón Rami-Porta por la revisión y sugerencias al trabajo.

\section{Referencias}

1. http://www.ine.cl/canales/chile_estadistico/censos_poblacion_vivienda/censo_pobl_vivi.php.

2. Moore EE, Malangoni MA, Cogbill TH, Shackford SR, Champion HR, Jurkovich GJ, et al. Organ injury scaling IV: Thoracic vascular, lung, cardiac, and diaphragm. J Trauma 1994;36:299-300.
3. Gómez G, Hola J. Trauma penetrante cardíaco en la unidad de emergencia del Hospital Carlos Van Buren. Rev Chil Cir. 2009;61:453-7.

4. Valenzuela M. Cancino P, Cabezas F. Experiencia en traumatismo torácico. Hospital Valparaíso. Rev Chil Cir. 2003;55;449-53.

5. Demirhan R, Onan B, Oz K, Halezeroglu S. Comprehensive analysis of 4205 patients with chest trauma: a 10-year experience. Interact Cardiovasc Thorac Surg. 2009;9:450-3.

6. Al-Koudmani I, Darwish B, Al-Kateb K, Taifour Y. Chest trauma experience over eleven-year period at al-mouassat university teaching hospital-Damascus: a retrospective review of 888 cases. J Cardiothorac Surg. 2012;7:35. doi: 10.1186/1749-8090-7-35.

7. Cook CC, Gleason TG. Great vessel and cardiac trauma. Surg Clin North Am. 2009;89:797-820.

8. Gómez G, Fecher A, Joy T, Pardo I, Jacobson L, Kemp $\mathrm{H}$. Optimizing outcomes in emergency room thoracotomy: a 20-year experience in an urban Level I trauma center. Am Surg. 2010;76:406-10.

9. Mollberg NM, Glenn C, John J, Wise SR, Sullivan R, Vafa A, et al. Appropriate use of emergency department thoracotomy: implications for the thoracic surgeon. Ann Thorac Surg. 2011;92:455-61.

10. Mollberg NM, Wise SR, De Hoyos AL, Lin FJ, Merlotti G, Massad MG. Chest computed tomography for penetrating thoracic trauma after normal screening chest roentgenogram. Ann Thorac Surg. 2012;93:1830-5.

11. O'Connor JV, Scalea TM. Penetrating thoracic great vessel injury: impact of admission hemodynamics and preoperative imaging. J Trauma 2010;68:834-7.

12. Monaghan SF, Swan KG. Tube thoracostomy: the struggle to the "standard of care". Ann Thorac Surg. 2008;86:2019-22.

13. Al-Koudmani I, Darwish B, Al-Kateb K, Taifour Y. Chest trauma experience over eleven-year period at al-mouassat university teaching hospital-Damascus: a retrospective review of 888 cases. J Cardiothorac Surg. 2012;7:35.

14. Demirhan R, Onan B, Oz K, Halezeroglu S. Comprehensive analysis of 4205 patients with chest trauma: a 10-year experience. Interact Cardiovasc Thorac Surg. 2009;9:450-3.

15. Ahmed N, Chung R. Role of early thoracoscopy for management of penetrating wounds of the chest. Am Surg. 2010;76:1236-9.

16. Goodman M, Lewis J, Guitron J, Reed M, Pritts T, Starnes S. Video-assisted thoracoscopic surgery for acute thoracic trauma. J Emerg Trauma Shock 2013;6:106-9.

17. Brundage SI. Systematic review and meta-analysis of antibiotic prophylaxis to prevent infections from chest drains in blunt and penetrating thoracic injuries. $\mathrm{Br} \mathrm{J}$ Surg 2012;99:513-4.

18. Mandal AK, Sanusi M. Penetrating chest wounds: 24 years experience. World J Surg. 2001;25:1145-9. 